\title{
三元合金异质结 $\operatorname{In}_{x} \mathrm{Ga}_{1-x} \mathrm{As} / \operatorname{In}_{x} \mathrm{Al}_{1-x} \mathrm{As}$ 的价带带阶 $\triangle \boldsymbol{E}_{\boldsymbol{v}}$ 值研究 ${ }^{*}$
}

\author{
郑金成 郑永梅 王仁智
}

(厦门大学物理系, 厦门 361005)

\section{关键词 异质结 价带带阶 平均键能方法}

三元合金异质结 $\mathrm{In}_{x} \mathrm{Ga}_{1-} \mathrm{As} / \mathrm{In}_{x} \mathrm{Al}_{1-} \mathrm{As}$ 是异质结器件的重要材料, 它广泛应用于微波 和光电器件中 ${ }^{[1]}$. 如组分 $x=0.3$ 的合金异质结可用于研制高电子迁移率晶体管 (HEMTs)、 绝缘椅场效应晶体管 (HIGFETs) 和谐振隧道二极管 (RTDs); 组分 $x=0.53$ 的三元合金异质 结 $\mathrm{In}_{x} \mathrm{Ga}_{1-} \mathrm{As} / \mathrm{In}_{x} \mathrm{Al}_{1-{ }_{x}} \mathrm{As}$ 广泛应用于光电子的高速光电器件中 ${ }^{[2]}$. 在决定量子阱、超晶格 电子态的因素中, 半导体异质结界面两侧价带带阶 $\Delta E_{\nu}$ 值 (即 valence-band offsets) 是重要的物 理量, 无论是理论计算还是实验研究都具有重要的意义.

对于由元素或化合物半导体构成的晶格匹配或失配类型的异质结的 $\Delta E_{\nu}$ 值, 已进行过相 当多的实验和理论计算研究 ${ }^{[3]}$. 但对于合金型异质结的 $\Delta E_{v}$ 值的理论计算研究工作还很少. 我们采用了 LMTO-ASA 能带计算方法, 并应用原子集团展开和平均键能结合的方法 ${ }^{[4]}$, 从理 论上研究了三元合金 $\operatorname{In}_{x} \mathrm{Ga}_{1-}{ }_{x} \mathrm{As} / \mathrm{In}_{x} \mathrm{Al}_{1-x} \mathrm{As}$ 异质结的 $\Delta E_{\nu}(x)$ 值, 发现计算结果与实验结 果相当符合, 说明平均键能与原子集团展开的方法适合于合金异质结的价带带阶 $\Delta E_{\nu}(x)$ 值 的理论计算.

\section{$1 \mathrm{In}_{l} \mathrm{Ga}_{4-l} \mathrm{As}_{4}$ 和 $\mathrm{In}_{l} \mathrm{Al}_{4-l} \mathrm{As}_{4}$ 有序结构平均键能 $E_{\mathrm{m}}$ 的研究}

本文采用基于局域密度泛函理论的线性 Muffin-Tin 轨道 (LMTO) 方法计算了三元合金

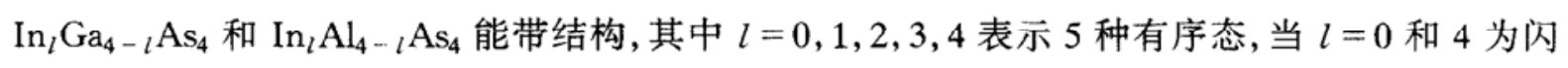
锌矿结构; 当 $l=2$ 时为 $L 1_{0}$ 结构; 当 $l=1$ 和 3 时为 $L 1_{2}$ 结构 ${ }^{[5]}$. 有序结构的晶格常数由 GaAs, AlAs 和 InAs 体材料的晶格常数按组分线性平均得到. 采用下式 ${ }^{[3,4]}$ 计算它们的成键 态能量 $E_{\mathrm{b}}$ 、反成键态能量 $E_{\mathrm{a}}$ 、平均键能 $E_{\mathrm{m}}$ :

$$
\begin{aligned}
& E_{\mathrm{b}}=\frac{1}{M N} \sum_{n=1}^{M} \sum_{k} E_{n}(k), \\
& E_{\mathrm{a}}=\frac{1}{M N} \sum_{n=M+1}^{2 M} \sum_{k} E_{n}(k), \\
& E_{\mathrm{m}}=\left(E_{\mathrm{b}}+E_{\mathrm{a}}\right) / 2,
\end{aligned}
$$


其中 $N$ 为晶体中原胞的个数, $M$ 为对应于成键态的价带数目或对应于反成键态的导带数目. 对于闪锌矿结构, $M=4$; 对于 $L 1_{0}$ 结构, $M=8$; 对于 $L 1_{2}$ 结构, $M=16$. 能带计算与 $E_{\mathrm{b}}, E_{\mathrm{a}}$ 和 $E_{\mathrm{m}}$ 计算中的 Brillou 区求和 (积分) 采用特殊 $K$ 点 ${ }^{[6]}$, 对于 $L 1_{2}$ 结构用 1 个特殊 $K$ 点; 对于 闪锌矿结构和 $L 1_{0}$ 结构用 2 个特殊 $K$ 点.

\section{2 三元合金 $\operatorname{In}_{x} \mathrm{Ga}_{1-x} \mathrm{As}$ 和 $\mathrm{In}_{l} \mathrm{Al}_{1-l} \mathrm{As}$ 的带阶参数 $E_{\mathrm{m}}(x)^{\circ}-E_{v}(x)$ 的计算}

在平均键能的带阶理论方法中, 异质结的带阶值主要决定于构成异质结体材料的带阶参 数 $E_{\mathrm{m}}-E_{\nu}$, 表 1 列出了 $\mathrm{In}_{l} \mathrm{Ga}_{4-l} \mathrm{As}_{4}$ 和 $\mathrm{In}_{l} \mathrm{Al}_{4-l} \mathrm{As}_{4}(l=0,1,2,3,4)$ 等 5 种有序结构的带阶参 数 $E_{\mathrm{m}}-E_{\nu}$ 的计算结果. 然后按原子集团展开的方法 ${ }^{[4]}$, 由下列式子得到 $\operatorname{In}_{x} \mathrm{Ga}_{1-}{ }_{x} \mathrm{As}$ 和 $\mathrm{In}_{x} \mathrm{Al}_{1-x} \mathrm{As}$ 合金的平均键能 $E_{\mathrm{m}}(x)$ 和价带顶 $E_{\nu}(x)$ 值, 即

$$
\begin{aligned}
& E_{\mathrm{m}}(x)=\sum_{l} P_{l}(x) E_{\mathrm{m}}^{l}, \\
& E_{\nu}(x)=\sum_{l} P_{l}(x) E_{\nu}^{l},
\end{aligned}
$$

其中统计权重为

$$
P_{l}(x)=\left(\begin{array}{l}
4 \\
l
\end{array}\right) x^{l}(1-x)^{4-l} .
$$

采用二次多项式拟合可以得到决定价带带阶的能带参数 $E_{\mathrm{m}}(x)-E_{\nu}(x)$ 值:

$$
\begin{aligned}
& \mathrm{In}_{x} \mathrm{Ga}_{1-x} \text { As: } E_{\mathrm{m}}(x)-E_{\nu}(x)=0.036 x^{2}+0.089 x-0.016, \\
& \operatorname{In}_{x} \mathrm{Al}_{1-x} \text { As: } E_{\mathrm{m}}(x)-E_{\nu}(x)=0.073 x^{2}-0.479 x+0.515 .
\end{aligned}
$$

由(7), (8)式拟合得到的结果也列于表 1 .

表 $1 \mathrm{In}_{l} \mathrm{Ga}_{4-l} \mathrm{As}_{4}$ 和 $\mathrm{In}_{l} \mathrm{Al}_{4-l} \mathrm{As}_{4}(l=0,1,2,3,4$ 等 5 种有序结构 $)$ 的带阶参数 $E_{\mathrm{m}}-E_{v}$ 和它们对应的 5 种合金成分的带阶参数 $E_{\mathrm{m}}(x)-E_{\nu}(x)$ 的计算结果 (单位: $\mathrm{eV}$ )

\begin{tabular}{ccccc}
\hline & \multicolumn{2}{c}{$\mathrm{In}_{l} \mathrm{Ga}_{4}-l \mathrm{As}_{4}$} & \multicolumn{2}{c}{$\mathrm{In}_{l} \mathrm{Al}_{4}-l \mathrm{As}_{4}$} \\
& $E_{\mathrm{m}}-E_{\nu}$ & $E_{\mathrm{m}}(x)-E_{v}(x)$ & $E_{\mathrm{m}}-E_{\nu}$ & $E_{\mathrm{m}}(x)-E_{\nu}(x)$ \\
\hline$l=0$ & -0.015 & -0.016 & 0.524 & 0.515 \\
$l=1$ & 0.005 & 0.008 & 0.401 & 0.400 \\
$l=2$ & 0.034 & 0.037 & 0.279 & 0.294 \\
$l=3$ & 0.071 & 0.071 & 0.198 & 0.197 \\
$l=4$ & 0.108 & 0.109 & 0.108 & 0.109 \\
\hline
\end{tabular}

\section{3 三元合金异质结 $\operatorname{In}_{x} \mathrm{Ga}_{1-} \mathrm{As} / \mathrm{In}_{x} \mathrm{Al}_{1-x} \mathrm{As}$ 的 $\triangle E_{v}(x)$ 值}

由(7)和(8)式的合金能带带阶参数的计算结果, 应用下式计算了合金异质结 $\mathrm{A} / \mathrm{B}$ 的价带 带阶:

$$
\Delta E_{\nu}(x)=\left[E_{\mathrm{m}}^{\mathrm{B}}(x)-E_{\nu}^{\mathrm{B}}(x)\right]-\left[E_{\mathrm{m}}^{\mathrm{A}}(x)-E_{\nu}^{\mathrm{A}}(x)\right] .
$$

用二次式表示为

$$
\triangle E_{\nu}(x)=0.037 x^{2}-0.567 x+0.531 \text {. }
$$

由上式可以看出, 三元合金异质结 $\operatorname{In}_{x} \mathrm{Ga}_{1-}{ }_{x} \mathrm{As} / \mathrm{In}_{x} \mathrm{Al}_{1-x} \mathrm{As}$ 的 $\Delta E_{\nu}(x)$ 值随着 $x$ 的增加而减 
小,也就是随着 $\mathrm{In}$ 组分的增加 (即 $\mathrm{Ga}$ 和 $\mathrm{Al}$ 组分的减少) 而减少. 从 (10)式可以看到, 表征 $\triangle E_{\nu}(x)$ 曲线弯曲程度的二次系数 (也称弯曲系数) 很小 (为 0.037 ), 表明 $\Delta E_{\nu}(x)$ 值随 $x$ 的 变化关系基本上是线性的, 说明三元合金异质结 $\operatorname{In}_{x} \mathrm{Ga}_{1-{ }_{x}} \mathrm{As} / \mathrm{In}_{x} \mathrm{Al}_{1-}$ As 的 $\triangle E_{\nu}(x)$ 值可以 由体材料 $\mathrm{GaAs}, \mathrm{AlAs}$ 和 InAs 的 $\Delta E_{\nu}$ 值按合金组分线性插值而得到 $\Delta E_{\nu}(x)$ 的近似值.

\section{4 计算结果与实验结果的比较}

我们把计算结果与实验结果作了比较, 表 2 列出了 $x=y$ 及 $x$ 接近于 $y$ 情况下的合金异 质结 $\triangle E_{\nu}(x, y)$ 的实验结果和本文的计算结果. 从表 2 可以看到理论计算结果与实验值相当 符合. 结果表明: 将平均键能与原子集团展开的方法结合起来, 形成了适合于合金异质结的价 带带阶 $\triangle E_{\nu}(x)$ 值的一种有效的理论计算方法.

表 2 三元合金异质结 $\mathrm{In}_{x} \mathrm{Ga}_{1-}{ }_{x} \mathrm{As} / \mathrm{In}_{y} \mathrm{Al}_{1-y} \mathrm{As}$ 的 $\Delta E_{\nu}$ 值与实验结果的比较 (单位: $\mathrm{eV}$ )

\begin{tabular}{|c|c|c|c|c|c|c|c|}
\hline & 本工作 & 文献[1] & 文献[1 $]^{a)}$ & 文献[2] & 文献 [7] & 文献[8] & 文献[9] \\
\hline$Q_{\mathrm{c}}\left(=\Delta E_{\mathrm{c}} / \Delta E_{\mathrm{g}}\right)$ & $0.656^{\mathrm{b})}$ & 0.66 & 0.62 & 0.68 & & 0.72 & 0.650 \\
\hline \multicolumn{8}{|l|}{$\Delta E_{v}(x, y)$} \\
\hline$(0,0)$ & 0.531 & & & & 0.55 & & \\
\hline$(0.3,0.29)$ & 0.368 & 0.36 & 0.41 & & & & \\
\hline$(0.3,0.3)$ & 0.364 & & & & & & \\
\hline$(0.52,0.52)$ & 0.246 & & & & & 0.22 & \\
\hline$(0.53,0.52)$ & 0.244 & & & 0.22 & & & \\
\hline
\end{tabular}

a) 数据转引自文献 $[1]$; b) 本文 $Q_{c}$ 的计算如下: 当 $(x, y)=(0.3,0.29)$ 时, $\Delta E_{\mathrm{g}}=1.07 ; \Delta E_{\mathrm{c}}=\Delta E_{\mathrm{g}}-\Delta E_{\mathrm{v}}=$ $0.702, Q_{\mathrm{c}}=\Delta E_{\mathrm{c}} / \Delta E_{\mathrm{g}}=0.656$

\section{参考文献}

1 Shieh J L, Chyi J I, Lin R J et al. Band offsets of $\mathrm{In}_{0.3} \mathrm{Ga}_{0.7} \mathrm{As} / \mathrm{In}_{0.29} \mathrm{Al}_{0.71}$ As heterojunction grown on GaAs substrate. Electron Lett, 1994, 30(25): $2172 \sim 2173$

2 Waldrop J R, Kraut E A, Farley C W et al. Measurement of $\operatorname{InP} / \mathrm{In}_{0.53} \mathrm{Ga}_{0.47} \mathrm{As}$ and $\mathrm{In}_{0.53} \mathrm{Ga}_{0.47} \mathrm{As} / \mathrm{In}_{0.52} \mathrm{Al}_{0.48} \mathrm{As}$ heterojunction band offset by X-ray photoemission spectroscopy. J Appl Phys, 1991, 69(1):372 378

3 王仁智,黄美纯. 异质结能带边不连续性的第一原理计算。中国科学, A 辑, 1992, (10): 1073

4 Wang Renzhi, Ke Sanhuang, Huang Meichun. Valence-band offset at $\mathrm{Al}_{x} \mathrm{Ga}_{1-x} \mathrm{As} / \mathrm{GaAs}$ : application of average-bond-energy theory in conjunction with the cluster expansion method. Phys Rev B, 1995, 52(3): 1935 1937

5 Lambrecht W R L, Segall B. Anomalous band-gap behavior and phase strability of c-BN-diamond alloys. Phys Rev B, 1993, $47: 9289 \sim 9296$

6 Chadi D J, Cohen M L. Special points in the Brillouin zone. Phys Rev B, 1973, 8:5 747 5 753

7 Batey J, Wright S L. Energy band alignment in GaAs: (Al, Ga) As heterostructures: the dependence on alloy composition. J Appl Phys, 1986, 59(1):200 209

8 Sugiyama $\mathrm{Y}$, Inata $\mathrm{T}$, Fujii $\mathrm{T}$ et al. Conduction band edge discontinuity of $\operatorname{In}_{0.53} \mathrm{Ga}_{0.47} \mathrm{As} / \mathrm{In}_{0.52}\left(\mathrm{Al}_{x} \mathrm{Ga}_{1-x}\right)_{0.48} \mathrm{As}$ heterostructures. Jpn J Appl Phys, 1986, 59: L648 L650

9. Kopf R F, Herman M H, Schnoes M L et al. Band offset determintion in analog graded parabolic and triangular quantum wells of GaAs/AlGaAs and GaAlAs/AlInAs. J Appl Phys, 1992, 71(10):5 004 5011 